\title{
Do the Global Lung Function Initiative 2012 equations fit my population?
}

\author{
To the Editor:
}

Knowing whether a patient's lung function result is similar to what can be expected of a healthy individual is critical for the correct interpretation of pulmonary function test results. Since lung function changes with growth and ageing and differs according to sex and ethnicity, there are now $>300$ published reference equations available for spirometry alone. Consequently, individual pulmonary function laboratories are left with a challenging decision: which reference equation do I choose? While it is recommended that reference equations are population specific, there are obvious logistical challenges to collect normative data from a large and representative population such that the resulting range of normal values are not biased.

Alternatively, laboratories can choose to use a published reference equation derived from a population similar to the one being tested. Several studies have shown that not all lung function reference equations produce the same results [1-3]. For instance, some equations are based on smaller samples of the population, while others are based on larger and representative population surveys. In some studies, spirometry curves are reviewed rigorously for quality control, while in others they are not. Some data are collected on antiquated equipment no longer available today, while others contain novel outcome measures. Furthermore, within each commercial spirometer, users have an overwhelming number of options: to use a single reference equation, or multiple reference equations combined into a single prediction set. The "stitching" of different equations results in artificial jumps in predicted values that could lead to erroneous interpretation of results and unnecessary intervention $[2,4,5]$. Most importantly, often the physicians interpreting results are unaware of which equations were used, and how the choice of equation might affect their interpretation [6-8]. Another common practice is to extrapolate reference equations beyond the age range of the intended age range, which has also been shown to bias interpretation of results [9].

The Global Lung Function Initiative (GLI) spirometry reference equations are one of the 300 available spirometry reference equation sets and come with their own advantages and disadvantages [10]. The GLI equations are based on data from $>74000$ individuals from 26 countries and are endorsed by six of the major respiratory societies. Despite the name, the world's largest populations, from the Indian subcontinent, Africa and Polynesian countries, are not represented. Nonetheless, the GLI dataset is the largest collection of normative spirometry data and reflects many populations, and the equations are a major step towards standardised interpretation of pulmonary function tests around the world. The distinct advantage of these equations is the large sample of healthy individuals across the age span (3-95 years) across multiple ethnic groups. The external validation of the reference equations is recommended by the American Thoracic Society/European Respiratory Society (ERS), and there have been many studies conducted that aim at validating the GLI equations. Several studies have found that the GLI equations fit the population well [11-15], including African children and adolescents of Bantu origin in Angola, Democratic Republic of Congo and Madagascar [16], while others have shown disagreement [17-19]. In some instances, the observed differences may be attributed to statistical differences that may not be clinically meaningful, while in others the observed differences may elucidate important population trends that may bias interpretation of results if the GLI equations are used inappropriately.

Any time a sample of the entire population is selected, there is a random chance that the selected sample will differ from the entire population; smaller samples are more likely to be different from the population. This can be illustrated as follows. A sample will have perfect fit if the mean calculated z-score using the GLI equations is 0 with a standard deviation of 1 , and $5 \%$ of observations are below the fifth percentile defined by a $\mathrm{z}$-score of -1.64 .

The large Health Survey for England (3661 males, mean \pm sD z-score for forced expiratory volume in $1 \mathrm{~s}$ (FEV1) of $0.04 \pm 1.03,4.89 \%$ of observations below fifth percentile), which was carried out on the same population as that used for the GLI equations, by the same staff, methods and quality control, provided an opportunity to assess the role of selection bias [20]. Random selection of 150 subsamples of 50-1500 records from the Health Survey for England datasets shows that the variability of the outcome decreased as the sample size increased (figure 1), the same pattern as that for the 58 datasets that made up the GLI study. However, the amplitude of differences is smaller for the Health Survey for England compared with the GLI dataset, possibly reflecting greater biological and technical differences in administering pulmonary function tests, as well as in quality control, in the 58 GLI datasets. It follows that it is unrealistic to expect that a sample will fit the overall population perfectly; even with large samples (150 and 1000 subjects), the 

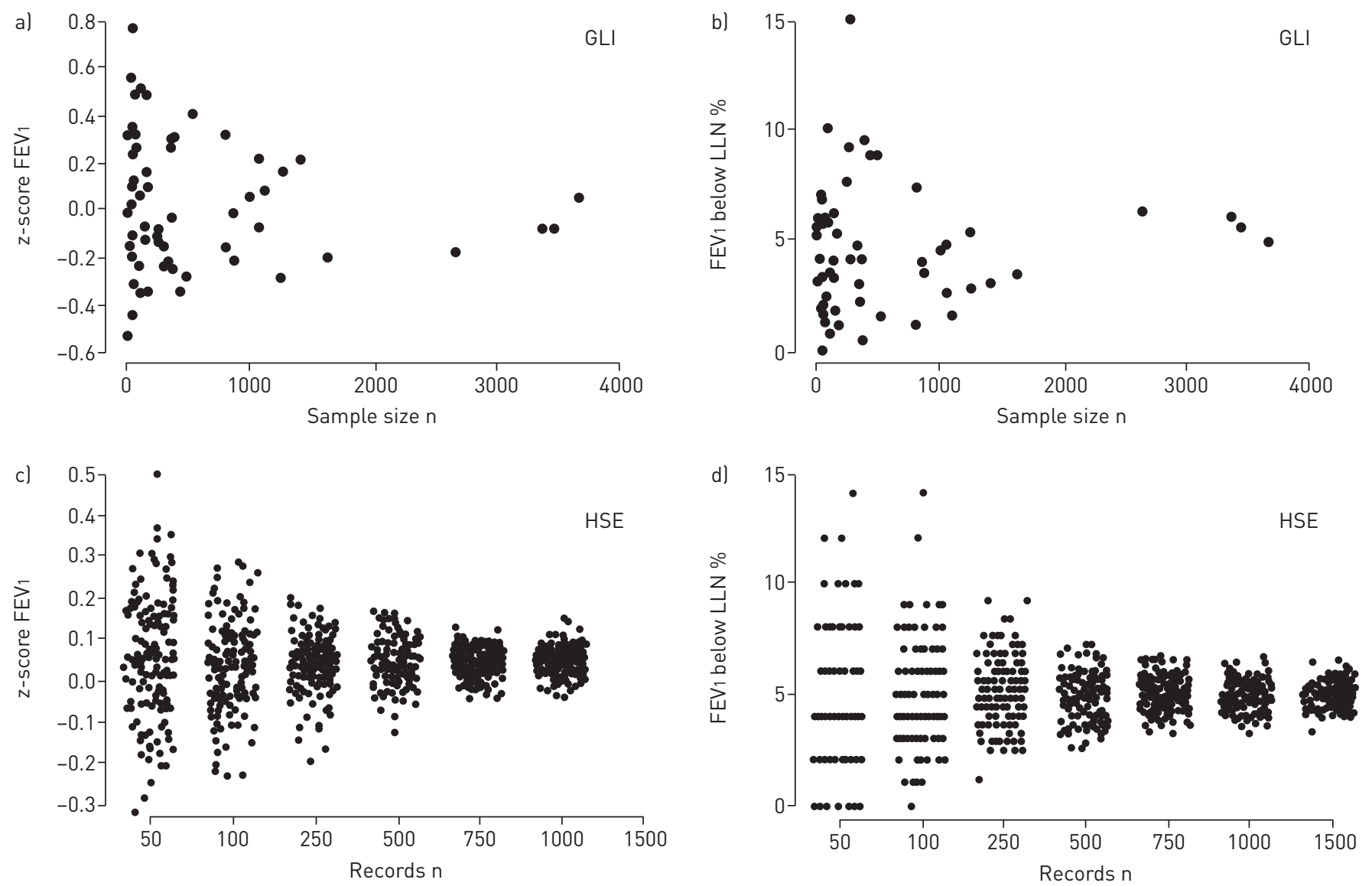

FIGURE $1 \mathrm{a}$ and b) Global Lung Function Initiative (GLI) predicted values for forced expiratory volume in $1 \mathrm{~s}$ (FEV 1 ) in males are based on 33487 data points from 58 datasets. The smaller the dataset, a) the larger the deviations from the GLI predicted values, and b) the more often the lower limit of normal (LLN) does not define the fifth percentile. $c$ and d) Using 150 randomly selected samples of 50-1500 records from the Health Survey for England (HSE; 3661 males) discloses the same sample size-related pattern.

average $z$-scores of the sample have been shown to differ up to 0.4 units from predicted within the same population, simply due to sampling variability [20].

Deciding whether the GLI, or any other reference equation, is appropriate for a particular population based on the observed differences of a selected sample of healthy individuals ought to be interpreted based on physiological and clinical significance as opposed to statistical significance. With a large enough sample, even the smallest differences can be statistically significant. What matters is whether an offset or trend reflects sampling error or true biological differences between the sample population and the reference population. The greatest emphasis should be placed on whether clinical decision making will be affected by the choice of reference equation. If differences persist and are biased in a systematic way, then elucidating the physiological reasons for the discrepancies between the GLI equations and these populations is imperative. For example, data collected as part of a large population survey in Japan showed that changes in socioeconomic and general health conditions were associated with changes in body frame, such as a change in relative leg length, which has been associated with a secular trend in lung function in Japan [19]. In view of genetic determinants of pulmonary function [21-23], it is similarly a challenge to define normal lung function in mixed-ethnicity populations; possibly this played a role in the finding of an unsatisfactory fit of the GLI equations in a Tunisian population where Berbers have sub-Saharan ancestry [22]. Improving our understanding of how social and cultural changes affect both somatic and lung growth will make an important contribution to the literature.

In studying whether there is a good fit of predicted values to a particular dataset, interpretation of data as a percentage of predicted should be discouraged. Using the percentage of predicted introduces an important age bias because the variance around the predicted value is not a fixed percentage of predicted $[10,24-26]$. Instead, data should be expressed as $\mathrm{z}$-scores, which indicate by how many standard deviations a measured value differs from the predicted, taking into account age, height, sex and ethnicity, and which are therefore unbiased. There are proportional differences in FEV1 and forced vital capacity 
(FVC) between ethnic groups, so that the FEV1/FVC ratio is for practical purposes the same in healthy populations [10]. A considerable difference from the predicted ratio (defined by z-scores) may therefore reflect limited sample size or a difference in health status.

In the event that the GLI equations do not fit a particular population, individual laboratories/countries are left with the options to revert to an older reference equation, or collect new normative data. In under-represented populations, the latter is highly advisable, both for our understanding of how lungs differ between different populations, but also to provide accurate normative data for these populations.

The GLI equations are not a permanent fixture, the network of collaborators was established to build an infrastructure to maintain and expand upon the data published in 2012. Individuals with data from under-represented populations, or with contemporary data collected from groups included in the GLI-2012 population, are encouraged to share their data with the GLI community (www.ers-education.org/guidelines/ global-lung-function-initiative.aspx). The ERS recently launched the ERS Research Agency [27], which will coordinate and fund respiratory research across Europe. The central aim of the Research Agency is to facilitate respiratory research through the coordination and support of the respiratory research community, and to assist in its efforts to obtain funding. The ERS Research Agency has recognised the GLI as a rich data source and now houses and maintains it. The dataset will soon be available not only to update the GLI-2012 equations but also for researchers to access for independent research questions.

There is no single available reference equation that can be applied universally to all pulmonary function laboratories globally. Each individual laboratory should carefully compare the options available for the population they are testing and choose the equations that are most appropriate. Importantly, laboratories should not use default equations arbitrarily without considering the implications and potential biases. Overall, there is an urgent need for the respiratory community to collect more normative data in under-represented populations to further improve how we interpret lung function. Regardless of which equations are used, clinical decisions should never be based solely on lung function test results. Results near the lower limit of normal should be cautiously interpreted and backed up with repeated testing and complementary laboratory clinical and physical findings.

○

@ERSpublications

No reference equation applies universally: default equations should not be used without considering potential biases http://ow.ly/29XB304sRrm

Philip H. Quanjer ${ }^{1}$ and Sanja Stanojevic ${ }^{2}$

${ }^{1}$ Dept of Pulmonary Diseases and Dept of Paediatrics-Pulmonary Diseases, Erasmus Medical Centre, Erasmus University, Rotterdam, The Netherlands. ${ }^{2}$ Division of Respiratory Medicine, Hospital for Sick Children, Toronto, ON, Canada.

Correspondence: Philip H. Quanjer. E-mail: pquanjer@gmail.com

Received: Sept 032016 | Accepted after revision: Sept 162016 | First published online: Nov 032016

Editorial comment in: Eur Respir J 2016; 48: 1535-1537.

Conflict of interest: None declared.

\section{References}

1 Rosenfeld M, Pepe MS, Longton G, et al. Effect of choice of reference equation on analysis of pulmonary function in cystic fibrosis patients. Pediatr Pulmonol 2001; 31: 227-237.

2 Stanojevic S, Stocks J, Bountziouka V, et al. The impact of switching to the new global lung function initiative equations on spirometry results in the UK CF registry. J Cyst Fibros 2014; 13: 319-327.

3 Subbarao P, Lebecque P, Corey M, et al. Comparison of spirometric reference values. Pediatr Pulmonol 2004; 37: 515-522.

4 Kirkby J, Aurora P, Spencer $\mathrm{H}$, et al. Stitching and switching: the impact of discontinuous lung function reference equations. Eur Respir J 2012; 39: 1256-1257.

5 Quanjer PH, Weiner DJ. Interpretative consequences of adopting the Global Lungs 2012 reference equations for spirometry for children and adolescents. Pediatr Pulmonol 2014; 49: 118-125.

6 Ghio AJ, Crapo RO, Elliott CG. Reference equations used to predict pulmonary function. Survey at institutions with respiratory disease training programs in the United States and Canada. Chest 1990; 97: 400-403.

7 Mohanka MR, McCarthy $\mathrm{K}$, Xu M, et al. A survey of practices of pulmonary function interpretation in laboratories in Northeast Ohio. Chest 2012; 141: 1040-1046.

8 Pattishall EN. Pulmonary function testing reference values and interpretations in pediatric training programs. Pediatrics 1990; 85: 768-773.

9 Linares-Perdomo O, Hegewald M, Collingridge DS, et al. Comparison of NHANES III and ERS/GLI 12 for airway obstruction classification and severity. Eur Respir J 2016; 48: 133-141.

10 Quanjer PH, Stanojevic S, Cole TJ, et al. Multi-ethnic reference values for spirometry for the 3-95-yr age range: the global lung function 2012 equations. Eur Respir J 2012; 40: 1324-1343.

11 Piccioni P, Tassinari R, Carosso A, et al. Lung function changes from childhood to adolescence: a seven-year follow-up study. BMC Pulm Med 2015; 15: 31. 
12 Hall GL, Thompson BR, Stanojevic S, et al. The Global Lung Initiative 2012 reference values reflect contemporary Australasian spirometry. Respirology 2012; 17: 1150-1151.

13 França DC, Camargos PA, Jones MH, et al. Prediction equations for spirometry in four- to six-year-old children. J Pediatr 2016; 92: 400-408.

14 Bonner R, Lum S, Stocks J, et al. Applicability of the Global Lung Function spirometry equations in contemporary multiethnic children. Am J Respir Crit Care Med 2013; 188: 515-516.

15 Hüls A, Krämer U, Stolz S, et al. Applicability of the Global Lung Initiative 2012 reference values for spirometry for longitudinal data of elderly women. PLoS One 2016; 11: e0157569.

16 Arigliani M, Canciani MC, Mottini G, et al. Evaluation of the Global Lung Initiative 2012 reference values for spirometry in African children. Am J Respir Crit Care Med 2016; in press [DOI: 10.1164/rccm.201604-0693OC].

17 Kainu A, Timonen KL, Toikka J, et al. Reference values of spirometry for Finnish adults. Clin Physiol Funct Imaging 2016; 36: 346-358.

18 Backman H, Lindberg A, Sovijärvi A, et al. Evaluation of the global lung function initiative 2012 reference values for spirometry in a Swedish population sample. BMC Pulm Med 2015; 15: 26.

19 Quanjer PH, Kubota M, Kobayashi H, et al. Secular changes in relative leg length confound height-based spirometric reference values. Chest 2015; 147: 792-797.

20 Quanjer PH, Stocks J, Cole TJ, et al. Influence of secular trends and sample size on reference equations for lung function tests. Eur Respir J 2011; 37: 658-664.

21 Menezes AM, Wehrmeister FC, Hartwig FP, et al. African ancestry, lung function and the effect of genetics. Eur Respir J 2015; 45: 1582-1589.

22 Ben Saad H, El Attar MN, Hadj Mabrouk K, et al. The recent multi-ethnic global lung initiative 2012 (GLI2012) reference values don't reflect contemporary adult's North African spirometry. Respir Med 2013; 107: $2000-2008$.

23 Kumar R, Seibold MA, Aldrich MC, et al. Genetic ancestry in lung-function predictions. N Engl J Med 2010; 363: 321-330.

24 Pellegrino R, Viegi G, Brusasco V, et al. Interpretative strategies for lung function tests. Eur Respir J 2005; 26: 948-968.

25 Sobol BJ, Sobol PG. Per cent of predicted as the limit of normal in pulmonary function testing: a statistically valid approach. Thorax 1979; 34: 1-3.

26 Miller MR, Pincock AC. Predicted values: how should we use them? Thorax 1988; 43: $265-267$.

27 Soriano JB, Paton J, Martin Burrieza F, et al. The ERS Research Agency: the beginning. Eur Respir J 2016; 47: 1017-1023.

\section{Viral mimic poly-(I:C) attenuates airway epithelial T-cell suppressive capacity: implications for asthma}

To the Editor:

In allergen-sensitised asthmatic individuals, allergen-specific type-2 T-helper cells proliferate and secrete type-2 cytokines (e.g. interleukin (IL)-4, -5 and -13), driving the airway inflammatory response that gives rise to the clinical symptoms of asthma. Both early-life sensitisation to aeroallergens and lower respiratory viral infections are important environmental risk factors for developing asthma. Additionally, respiratory viral infections are the most common trigger for asthma exacerbations. Of interest, many asthma susceptibility genes are expressed in the airway epithelium [1], which forms the first continuous line of defence against inhaled environmental insults, including viruses and aeroallergens. Impaired immune regulation and failure to maintain tolerance to allergens is thought to contribute to allergic sensitisation. Asthma epithelium may be deficient in its innate immune defence against viral infections, resulting in increased viral replication upon rhinovirus infection compared to nonasthma-derived epithelial cultures [2]. Furthermore, there is evidence for loss of the mucosal immune barrier in asthma, with disruption of epithelial integrity $[1,3]$. This may lead not only to increased permeability, but also to the release of pro-inflammatory mediators, specifically of cytokines that drive type-2 responses $[3,4]$. We recently observed that the ability of allergens to disrupt epithelial barrier function is related to the development of type-2-mediated inflammation in asthma [5, 6]. Furthermore, we demonstrated that healthy murine lung epithelium is a potent inhibitor of T-cell proliferation and that this inhibition is lost upon viral infection [7]. It is unknown if this immune regulatory effect is displayed by human epithelium and is dysregulated in asthma. We hypothesise that changes in this regulatory effect translate into aberrant regulation of T-cell responses in asthma. We studied the epithelial regulation of T-cell proliferation and cytokine responses upon epithelial stimulation with a viral mimic, using co-culture of human T-cells and primary bronchial epithelial cells (PBECs) from healthy controls and asthma patients. 